\title{
Desafíos para alcanzar universalidad con equidad en salud en América Latina en el siglo XXI*
}

\author{
Challenges to universality with health equity in Latin America \\ in the 21st century
}

\section{Julio Manuel Suárez Jiménez}

Máster en Salud Pública. Oficina Panamericana para la Salud/Organización Mundial para la Salud. Santiago de Chile, Chile.

Los valores de universalidad y equidad en salud tienen aceptación prácticamente generalizada en el mundo de hoy. Contar con sistemas de salud que atienda a todos los ciudadanos sin distinción de clase social, raza, credo, género u otras condiciones, es requisito para una vida colectiva digna. Estos valores están consagrados en la mayoría de los textos constitucionales de los países y han sido ratificados por resoluciones de Organización Panamericana de la Salud (OPS) y la Organización Mundial de Salud (OMS). ${ }^{1,2}$

La OPS estima que en América Latina y el Caribe alrededor de 230 millones de personas (46\%) no cuenta con seguro de salud. ${ }^{3}$ Según la CEPAL, la tasa de informalidad laboral en la Región de Las Américas es de 52 \% a pesar de los avances en este sentido en años anteriores hoy amenazados por las crisis económicas. ${ }^{4}$ Es decir, más de la mitad de los trabajadores de la Región no tiene empleo formal y por tanto carece de protección de la Seguridad Social, quedando automáticamente excluidos de la protección en salud por esta vía.

El gasto mínimo necesario por persona y por año para prever servicios "básicos" de salud es de 35 a 50 dólares de los Estados Unidos según la OMS, pero 64 países no alcanzaban esta cifra en el 2004, uno de ellos es Haití. ${ }^{5}$ Cuatro países de América Latina: Bolivia, Honduras, Nicaragua y Paraguay tenían en 2005 un gasto percápita anual en salud menor a 100 USD. ${ }^{6}$ 
América Latina no es la Región más pobre del mundo, pero es la más desigual en la distribución de su riqueza. Mientras el $10 \%$ más rico de la población concentra alrededor del $35 \%$ de los ingresos, el $40 \%$ más pobre recibe alrededor del $15 \%$, con los valores aún inferiores en Bolivia y República Dominicana, en los que los más pobres solo captan cerca del $11 \%$ de los ingresos. ${ }^{7} \mathrm{El}$ ingreso medio por persona en los hogares del quintil más rico de Las Américas supera 20 veces el del quintil más pobre, siendo esta diferencia aún mayor en algunos países como Bolivia, Haití y Paraguay donde la razón es de 42,$3 ; 26,6$; y 25,7 respectivamente. ${ }^{8}$ En contraste, la misma razón de ingresos en Canadá, país de La Región con un sistema universal de salud, es de $5,5 .^{6}$

La población envejece aceleradamente y crece la epidemia de enfermedades crónicas y daños asociados a la violencia. Ello representa un importante retos para nuestras sociedades por el cambio de la pirámide demográfica, el aumento de la carga de enfermedades crónicas y las contradicciones que esta situación presenta a la organización tradicional del trabajo, la seguridad social y los servicios de salud, pensados para una población más joven en la que prevalece problemas y condiciones agudas.

Según datos citados por la OMS en el informe Sobre la Salud en el Mundo 2008, en la región de Europa el $9 \%$ de la población es partidaria de una cobertura sanitaria integral y en los Estados Unidos, que no cuenta con un sistema nacional de seguro de enfermedad, más del $8 \%$ está a favor de ella. ${ }^{9}$ Este informe identifica un grupo de problemas o contradicciones relacionadas con los sistemas de salud a los que recomienda enfrentar con procesos de reforma. Los problemas aparecen en el siguiente recuadro tomado del informe citado (Recuadro 1 ).

Recuadro 1. Informe sobre la Salud en el Mundo 2008

Atención de salud inversa. El gasto de salud pública tiende a beneficiar a los ricos más
que a los pobres.
Atención a la salud empobrecedora. Más de 100 millones de personas al año tiene
que costear de su propio bolsillo los gastos de atención de salud y, como consecuencia,
muchas veces caen en la pobreza.
Atención fragmentada. Redes de servicios fragmentada; especialización excesiva de
los médicos; enfoque limitado de programas de control de enfermedades; gobernanza
basada en la intervención o laissez-faire, lo que permite que prospere la atendón de
carácter comercial no regulada; ayuda internacional fragmentada.
Atención de salud con riesgos. No se aumplen adecuadamente las nomas de
seguridad e higiene, tasas elevadas de infecciones nosocomiales, errores con los
medicamentos y otros sucesos adversos.
Atención mal dirigida. Reoursos concentrados en servicios curativos en detrimento de
laprevención primaria y la promodón de la salud (se podría evitar hasta el $70 \%$ de las
enfermedades y las muertes).

El ideal de universalidad con equidad ha sido llevado a la práctica en un grupo de países, tanto desarrollados como en vías de desarrollo, con diferentes modelos. En general, los países que cuentan con sistemas universales obtienen mejores resultados en salud y su distribución social es más equitativa que en aquellos que no cuentan con sistema de salud que protejan a todos sus ciudadanos. ${ }^{10}$ Estos países han logrado el aseguramiento universal mediante sistemas nacionales de servicios de salud a los que se accede por derecho ciudadano sin mediar un seguro 
propiamente dicho, o mediante sistemas de seguros públicos con cobertura efectiva universal. La otra cara de la moneda son los países que aún mantiene sistemas segmentados y fragmentados, que por su propia esencia excluyen a una parte de la población de cualquier acceso a los servicios y discriminan a los que logran algún acceso en función de su pertenencia a uno u otro segmento social. Este es el caso de una buena parte de los países de América Latina.

La riqueza económica de los países es un factor que puede favorecer la protección social universal en salud cuando los países dedican una parte apropiada de esta para proteger a sus ciudadanos a través de un modelo de aseguramiento universal. Sin embargo, no basta la riqueza para lograr universalidad y mucho menos la equidad. Es esencial que cada sociedad defienda su sistema de salud y los recursos destinados a este como un bien público apreciado para evitar que las clases políticas coloquen a la salud como última prioridad o, aún, como ninguna. Defender un presupuesto público suficiente -también para educación y cuidado del ambientees defender el futuro y la viabilidad de nuestras naciones. Cada sociedad debe decidir que tipo de sistema de salud quiere tener, qué porción de su riqueza nacional está dispuesta a dedicar al sistema y los servicios de salud y cómo gestionará dicho sistema. No obstante, en la práctica estas decisiones quedan en manos de los grupos que detentan el poder formal y la participación social en ellas es limitada o nula en la mayoría de los países.

\section{DESAFÍOS}

Para alcanzar los objetivos de universalidad con equidad, los sistemas de salud de América Latina deben enfrentar y resolver un grupo de desafíos comunes a la mayoría de estos. A los desafíos de orden social, político y económico ante mencionados, se suman otros directamente relacionados a la naturaleza de los sistemas de salud. Sin pretender agotar la lista, los que aparecen a continuación son cardinales actualmente.

\section{Estructura y gestión}

El problema mayor es la segmentación y fragmentación de los sistemas de salud. La división de los sistemas de salud en subsistemas paralelos e incomunicados genera en sí mismo iniquidad al clasificar a los usuarios de acuerdo con su pertenencia a uno u otro segmente. Junto al segmento privado presente con mayor o menor fuerza en casi todos los países, típicamente se identifica dos grandes segmentos públicos: el segmento de los que están protegidos por la Seguridad Social y el segmento de los que no tiene dicha protección. Este último constituye más del $50 \%$ de la población en varios países y habitualmente queda a cargo de los Ministerios de Salud o sus equivalentes, que a través de servicios propios, de estados o provincias y municipios, complementados por hospitales privados -con o sin fines de lucro- brinda atención generalmente pobre para pobres. Segmentación y fragmentación agregan un problema de marco ineficiencia al multiplicar los costos de transacción e infraestructura pública. De esta forma quedan lesionadas desde el origen la universalidad y la equidad.

En varios países de La Región de Las Américas y El Caribe, la rectoría y gestión de los sistemas y los servicios de salud es débil, burocrática, ineficiente y poco respetada por los beneficiarios y usuarios de los sistemas. En los sistemas segmentados y fragmentados esta realidad se expresa con mayor fuerza y es más difícil de superar. Estos sistemas tienen serias dificultades para organizar redes integradas de servicios de salud, condición imprescindible para brindar una atención 
continuada, integral y de calidad. La OPS ha lanzado una iniciativa para impulsar la construcción y desarrollo de Redes Integrales de Servicios de Salud (RISS) basadas en la Estrategia de Atención Primaria de la salud. ${ }^{11}$ El propósito de la iniciativa de la OPS sobre RISS es contribuir al desarrollo de Sistemas de Salud basados en la asistencia primaria de salud (APS) en los que se preste servicios que sean más accesibles, equitativos, eficientes, de mejor calidad técnica y que satisfagan mejor las expectativas de los ciudadanos. La OPS considera que las RISS son una de las principales expresiones operativas del enfoque de la APS a nivel de los servicios de salud, que contribuye a hacer realidad varios de sus elementos esenciales tales como la cobertura y el acceso universal, el primer contacto, la práctica atención integral, integrada y continua; el cuidado apropiado, la organización y gestión óptimas y la acción intersectorial, entre otros.

En el recuadro 2 se aprecian los atributos de la RISS.

Recuadro 2. Atributos esenciales de las Redes Integrales de Servicios de Salud

1. Población/territorio a cargo definido y amplio conocimiento de sus necesidades y preferencia en salud, las cuales determinan la oferta de servicios de salud.

2. Una extensa red de establecimientos de salud que comprende todos los niveles de atención y que provee tanto servicios de salud personales como de salud pública.

3. Un 1er. nivel de atención que actúa como puerta de entrada al sistema, que integra y coordina el cuidado de la salud, y que resuelve la mayoría de las necesidades de salud de la población.

4. Prestación de servicios de especialidad en el lugar más apropiado, los cuales se dan preferentemente en ambientes extra-hospitalarios.

5. Existencia de mecanismos de coordinación asistencial a lo largo de todo el continuo de servicios de salud.

6. Cuidado de la salud centrado en la persona, la familia y la comunidad/territorio.

7. Un sistema de gobernanza único para toda la red.

8. Participación social amplia.

9. Gestión integrada de los sistemas de apoyo administrativo, clínico y logístico.

10. Recursos humanos suficientes, competentes, comprometidos y valorados por la red.

11. Sistema de información integrado y que vincula a todos los miembros de la red.

12. Financiamiento adecuado e incentivos financieros alineados con las metas de la red.

13. Acción intersectorial.

La gestión centralizada de algunos sistemas impide la toma de decisiones donde se producen los problemas de salud y se implementa una buena parte de las soluciones. Se limita la iniciativa de los gerentes de sistemas locales y la intersectorialidad, y se cercena la participación de los verdaderos dueños de los sistemas públicos, la población. De otro lado, la descentralización puede ser un problema cuando el sistema no está estructurado sobre valores y principios compartidos y carece de una buena rectoría, coordinación y gestión en función de necesidades y no de los intereses de los prestadores de servicios y su capacidad de oferta. 


\section{Desempeño de las funciones esenciales de salud pública}

Los países que logran buenos resultados en salud desempeñan con éxito un grupo de funciones estratégicas de responsabilidad pública en cuyo cumplimiento los sistemas de salud tienen un papel central. La "Iniciativa Salud Pública en Las Américas"lanzada por la OPS definió 11 Funciones Esenciales de Salud Pública (FESP). ${ }^{12}$ En las evaluaciones de las FESP realizadas a nivel regional y en los países, se demuestra que varias de estas funciones tienen un desempeño deficiente (figura 1). Siendo todas ellas "esenciales" para el desarrollo de la salud, el buen desempeño de los sistemas de salud en el cumplimiento de estas funciones es parte integral de los esfuerzos para lograr universalidad con equidad. ${ }^{12,13}$

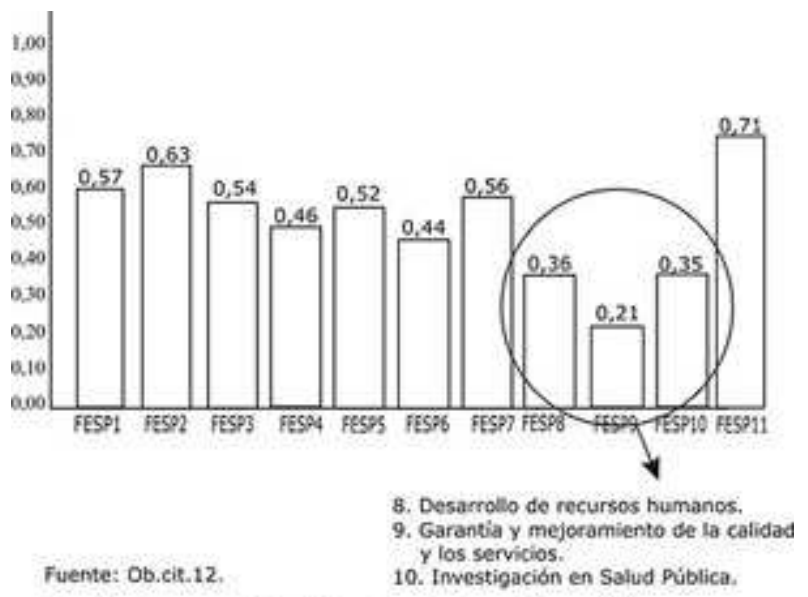

Fig. 1. Desempeño de las Funciones Esenciales de Salud Pública(FESP)en la Región de Las Américas.

\section{Modelo de atención}

Para alcanzar universalidad con equidad resulta decisivo el modelo de atención que se implementa en el sistema de salud. En una buena parte de nuestros países, el modelo predominante está basado en el hospital y en el predominio de la atención médica curativa a eventos agudos en unidades aisladas. Las evidencias acumuladas indican que este modelo de atención no puede atender efectiva y eficientemente las necesidades de la población y mucho menos de equidad. Se requiere entonces reorientar el modelo de atención hacia uno cimentado en los principios y valores de la Estrategia de Atención Primaria lanzada hace 40 años en Alma Atá y renovada recientemente por OMS y OPS. ${ }^{10}$ Los sistemas de salud deben priorizar la promoción de la salud y prevención de riesgos y daños, construirse a partir de un primer nivel de atención resolutivo y de calidad, y organizarse en unidades y equipos de salud multiprofesionales estructurados en una red integral que cubra todas las necesidades de atención, desde el trabajo con las personas sanas hasta la curación y rehabilitación priorizando las condiciones crónicas de mayor prevalencia que requieren una atención longitudinal en RISS. Solo así será posible enfrentar los desafíos sociales, demográficos, epidemiológicos en pro de la universalidad con equidad. En esta misma dirección se inscribe la iniciativa de OPS/OMS antes citada sobre RISS, inscrita en el proceso de renovación de la Estrategia de Atención Primaria.

Otra dimensión esencial en el modelo de atención es la calidad. Colocar acceso y calidad como metas secuenciales es erróneo. Una buena calidad de la atención es parte integrante inseparable del acceso a los servicios. Extender coberturas y acceso a servicios de baja calidad para pobres es éticamente injustificable. Iniciativas de OPS/OMS como "Manos Limpias" y "Cirugía Segura" en el marco de la 
"Alianza Mundial por la Seguridad del Paciente" son buenos ejemplos a seguir en esta dimensión. ${ }^{14}$

\section{Recursos humanos}

Para lograr universalidad con equidad es imprescindible contar con personal de salud suficiente, distribuido equitativamente a lo largo de la red de servicios, con la calificación y motivación necesarias para desempeñar su trabajo. Resolver esta ecuación es uno de los mayores desafíos -sino el mayor- para lograr los objetivos de universalidad y equidad en salud.

En la evaluación regional del desempeño de las FESP citadas anteriormente, la función "desarrollo de recursos humanos y capacitación en salud pública" quedó entre las tres que obtuvieron las calificaciones más bajas. Esta baja calificación se repite en la mayoría de las evaluaciones de la FESP realizadas a nivel nacional y subnacional en varios países.

La OMS ha calculado que 57 países (más de $40 \%$ de la población mundial) no cuentan con suficientes trabajadores de salud para proporcionar el nivel mínimo de atención requerido. En Las Américas, al aplicar la norma de densidad de recursos humano que la OMS ha fijado como valor básico mínimo ( 25 trabajadores de salud por 10000 habitantes), 15 países están por debajo, y aunque este grupo de naciones concentra a 19,5\% de la población de la región, tiene solo $11,5 \%$ de los médicos y $3,1 \%$ de las enfermeras. Se requerirían otros 128000 médicos y enfermeras para elevar la razón de densidad de recursos humanos de toda la región hasta el nivel de $25 .^{15}$

El problema se agrava con la proliferación de instituciones y cursos universitarios en los que se forman los profesionales y técnicos de salud, en contextos nacionales que regulan deficientemente dicha formación dejando espacio a la mala calidad, con planes de estudio que no responden a las necesidades de los sistemas de salud y los problemas prevalentes y siguen orientados hacia la labor hospitalaria y curativa.

Por otra parte, es importante reconocer la importancia que tiene en esta esfera la posición y acciones de los sindicatos de trabajadores, el sector patronal y las asociaciones profesionales. Estos pueden ser factores favorables o restrictivos para los procesos de desarrollo de los sistemas y los servicios integrados de salud que deben ser abordados con especial cuidado.

\section{Financiamiento}

El gasto público en salud es un buen indicador del compromiso con la salud. A pesar de las evidencias a favor de invertir en los sistemas de salud por la relación sinérgica entre estos, la salud de la población, el bienestar y la riqueza de las naciones, ${ }^{16}$ en muchos países, especialmente en los de menor desarrollo económico, la inversión en salud es insuficiente. De 70 países de "Desarrollo Humano Alto" según el Índice de Desarrollo Humano (IDH) del más reciente informe del PNUD, ${ }^{8}$ solo 9 tienen un gasto privado superior al público. El gasto público en salud en los 20 países con mayor IDH es de 6,5 \% o más del producto interno bruto (PIB). En todos ellos el gasto público es proporcionalmente mayor que el privado excepto en los Estados Unidos de América y en 16 países el gasto público representa el $70 \%$ o más del gasto total en salud. ${ }^{6}$ Esta relación se invierte paradójicamente en la mayoría de los países de "Desarrollo Humano Bajo", entre los que predomina el gasto privado sobre el público. 
El gasto de bolsillo, considerado el más regresivo, es aún muy alto especialmente en la adquisición de medicamentos. En algunos países, el gasto de bolsillo en salud llega a ser más del $95 \%$ de todo el gasto privado, el gasto en medicamentos supera el $60 \%$ del gasto total de bolsillo y familias enteras caen en la pobreza por tener que asumir privadamente el pago de los servicios de salud. En este sentido, el antes citado Informe sobre la Salud en el Mundo 2008, lanzado por la OMS dedicado a la atención primaria de la salud dice lo siguiente:

Transcurrido más de 30 años desde la Declaración de Alma Atá, que representó una señal de alarma apremiante en pro de la equidad sanitaria, la mayor parte de los sistemas de atención sanitaria del mundo siguen dependiendo del método menos equitativo de financiación de los servicios de salud: los pagos directos que las personas enfermas o sus familias efectúan en el punto de prestación de la atención. Para 5600 millones de personas de los países de ingresos bajos y medios, más de la mitad de todos los gastos médicos salen de su propio bolsillo. Este sistema priva a muchas familias de la atención que necesitan porque no pueden permitírsela. Además, todos los años más de 100 millones de personas se ven abocadas a la pobreza en todo el mundo de resultas de unos gastos médicos catastróficos.

Las crisis económicas y financieras como la que atravesamos en el 2009 se reflejan inevitablemente en la cantidad y calidad del gasto en salud y no es de extrañar que ocurran recortes en el presupuesto público destinado a la salud. Hay un déficit acumulado de dicho gasto y para lograr los objetivos de universalidad con equidad se debe incrementar el gasto en salud. De ahí que el contexto actual es más de amenazas que de oportunidades. En un informe de enero del presente año, la CEPAL avizora un aumento de la pobreza en La Región de Las Américas debido al incremento de los precios de los alimentos y la energía y el deterioro del mercado laboral y dice que el empleo será la variable de ajuste. ${ }^{4}$ Podría añadirse el alto precio de los medicamentos como otro factor que en el escenario descrito por CEPAL agravaría la pobreza y el acceso a la protección y atención de la salud.

En un reciente articulo, la Directora de la OPS Dra. Mirta Roses alerta sobre posibles consecuencias de la actual crisis económica, como el incremento de la pobreza, la desnutrición y la exclusión en el acceso a los servicios básicos lo que empeoraría la desigualdad que históricamente afronta la Región. Nos avisa sobre el riesgo de que se reduzca el personal o el presupuesto asignado a los servicios públicos de salud por la posible reducción de ingresos fiscales, con sus negativas consecuencias en el capital humano acumulado, lo que haría aún más difícil salir de la crisis y, luego, subsanar sus efectos. Lo que se debe hacer, nos dice la Dra. Roses, es "invertir en salud para proteger los logros y minimizar los efectos sociales de la crisis tanto a corto como a largo plazo, y además trabajar para que ese gasto público ayude a minimizar la desigualdad." ${ }^{17}$

\section{Medicamentos y tecnologías sanitarias}

El acceso a los servicios de salud implica el acceso a medicamentos y tecnologías diagnósticas y terapéuticas. Sabemos que la falta de acceso a medicamentos y tecnologías, por motivos económicos, culturales y geográficos es un problema mayor en muchos sistemas de salud. Especialmente en relación con los medicamentos, el hecho de que su adquisición dependa mayoritariamente de gasto de bolsillo limita a veces seriamente una atención completa e integral. Los medicamentos representan entre el 20 y el $60 \%$ del gasto en salud en los países en desarrollo, ${ }^{18}$ y cerca del $90 \%$ de la población compra directamente medicamentos de sus bolsillos constituyendo este el mayor gasto de las familias después de los alimentos. ${ }^{19}$ Por otra parte, más del $90 \%$ de la producción de 
medicamentos esta concentrada en un pequeño grupo de países desarrollados y el valor de los medicamentos tiende a crecer. ${ }^{18}$

Adicionalmente, la ubicación de tecnologías de alta complejidad diagnóstica y terapéutica como la imaginología computarizada, hemodiálisis, radioterapia y otras, habitualmente se concentra en los medianos y grandes conglomerados urbanos, lo que dificulta el acceso de quienes viven en zonas rurales o apartadas por razones de comunicación y costo. De otra parte, la concentración espacial de estas tecnologías agrega un problema de marco ineficiencia del sistema al ser redundantes en algunos lugares y ausentes en otros. La carencia de verdaderas RISS que garanticen el acceso a los servicios en todos los niveles organizativos y a todo el abanico de prestaciones, desde la promoción de la salud hasta la rehabilitación, agrava las dificultades de acceso a medicamentos y tecnologías.

\section{Participación social y rendición de cuentas}

El sistema público de salud de cualquier país pertenece en primer instancia a su población. Se ha logrado avanzar en las últimas décadas en espacios y modalidades de participación social pero aún falta mucho por hacer. La creación y funcionamiento en varios países de consejos de salud en las diferentes esferas de gestión (nacional, provincial, municipal, jurisdiccional...) son buenos ejemplos de participación social en los sistemas de salud. También son buenos ejemplos los comités de gestión de hospitales y centros de salud que incluyen representantes de la comunidad, las organizaciones de usuarios, los grupos de interés aglutinados por padecimientos específicos, redes en defensa de los pacientes y otras variantes de participación. Sin embargo, mucho camino hay por recorrer en relación con la transparencia y rendición de cuentas sobre el uso de los recursos públicos, la información oportuna y apropiada a los usuarios sobre opciones de atención, el acompañamiento por el enfermo y sus familiares del proceso clínico, y la adecuación de los servicios a las necesidades e intereses de la población a la que sirve, entre otros ámbitos de acción.

\section{HACIA SISTEMAS UNIVERSALES DE SALUD EN EL SIGLO XXI}

Construir sistemas universales de salud requiere un consenso de la sociedad que coloque a la salud y sus determinantes en el centro de las prioridades nacionales. Ello significa alcanzar un nuevo pacto social que dedique las mayores energías a mejorar las condiciones y calidad de vida, atendiendo integralmente -no nominalmente- las necesidades de salud, educación, alimentación, seguridad social y cuidado del ambiente, como base del crecimiento económico sostenible y no como limosna de la riqueza del país.

El pacto social deberá establecer una agenda estratégica de salud que contemple entre sus objetivos -aunque únicamente- enfrentar los desafíos identificados anteriormente:

- La reforma del sistema de salud y su gestión sobre valores y principios presididos por la universalidad y la equidad;

- fortalecimiento de las funciones esenciales de salud pública;

- orientación del modelo de atención hacia y desde la atención primaria;

- garantía del personal de salud suficiente y calificado para trabajar en la red de servicios con énfasis en la capacidad resolutiva del primer nivel de atención; 
- garantía del financiamiento público suficiente para sostener un sistema universal y creación de bases y condiciones para la gestión eficiente de recursos;

- regulación y garantía del acceso a medicamentos y tecnologías sanitarias de calidad incorporadas mediante procesos de evaluación sanitaria y

- fortalecer la participación y le control social del sistema de salud y los servicios.

La agenda estratégica se basa en valores y principios y tiene tres espacios de acción. La figura 2 resume la idea utilizando simbólicamente la rosa de los vientos, guía de marinos, para no perder el rumbo.

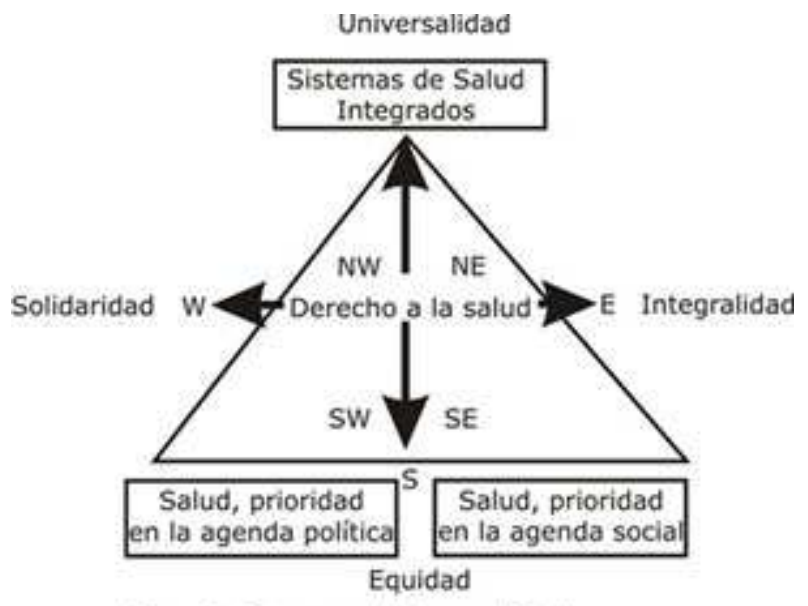

Fig. 2. Esquema Universalidad.

En el centro deberá estar el derecho a la salud cimentado en los valores de universalidad, equidad, integralidad y solidaridad. En el documento sobre la Renovación de la APS aprobado por los Ministros de los países miembros de la OPS, se expresa esta perspectiva como el "derecho a alcanzar el grado máximo de salud posible", "con la mayor equidad y solidaridad", considerando la necesidad de "dar respuesta a la necesidades de salud de la población". Este ideal se concreta mediante la protección colectiva universal a través de acciones de salud pública y la garantía de acceso oportuno de las personas a servicios de salud de calidad sin discriminación de cualquier origen.

Se identifican tres espacios estratégicos de acción sin que el orden implique mayor o menor importancia entre ellas.

\section{Sistemas de salud integrados}

En primer lugar la construcción y desarrollo de sistemas de salud integrados basados en la APS. Para ello, el desarrollo de RISS es un elemento clave. Se trata de sistemas de salud que superan la segmentación y fragmentación al colocar en primer plano el derecho ciudadano a la salud a partir de sus necesidades, y subordinar a este objetivo la oferta de servicios y los intereses particulares de las instituciones y actores sociales que participan en el sistema. Se requiere fortalecer la gobernanza del sistema, la gestión de las redes integradas, los mecanismos de recolección y asignación de fondos para la salud, el desarrollo de los recursos humanos del sistema, los sistemas de información en función de los procesos decisorios, la planificación estratégica y la política de medicamentos entre otros elementos sustantivos.

\section{Salud prioridad en la agenda política}


La construcción de sistemas integrados de salud es un proceso eminentemente político en el que participan varios actores y fuerzas sociales amalgamados por el objetivo común. En la mayoría de los casos, se trata de un proceso de mediano y largo plazo, conflictivo y complejo. La existencia en cada país de fuerzas sociales con capacidad de liderar el proceso es un factor decisivo. Actores significativos son las instituciones del sector de la salud, universidades, institutos de investigación, los trabajadores de la salud y los usuarios organizados, que utilizan su poder y capacidad de movilizar voluntades de intereses en función del proceso. La prioridad que al final tenga salud en la agenda política de cada país se define en espacios y procesos en general ajenos al sector de la salud. La decisión sobre la asignación de los recursos destinados a la salud depende de un proceso de debates y lobbies entre múltiples fuerzas y actores sociales interesados. Generalmente existen intereses opuestos que luchan por incrementar sus presupuestos propios. Si salud no es prioridad en este espacio, es prácticamente imposible que lo sea la protección universal y equitativa.

\section{Salud prioridad en la agenda social}

Por último, colocar a la salud como prioridad para la sociedad. Se trata de eliminar o disminuir la distancia entre sistema de salud y sociedad, entre gestores de los servicios y quienes los usan, entre prioridades y necesidades, entre demanda y oferta. No es suficientemente común que los ciudadanos estén orgullosos y satisfechos de sus sistemas públicos de salud y los defiendan como un valor irrenunciable de su sociedad. Se trata de una pertenencia de doble vía. De un lado, cuánto una sociedad aprecia, se apropia y defiende como conquista su sistema de salud. De otro lado, cuánto un sistema de salud responde a la necesidades de su población y tiene a esta en el centro de su quehacer. Dicho de otra forma, cuánto quieren y aprecian a su sistema de salud la población y sus representantes y cuán sensible y consecuente es el sistema de salud con su sociedad.

El sentido de pertenencia debe reflejarse en las políticas prioritarias de país más allá de las fronteras de los sistemas. El peso específico de la salud y su prioridad en la agenda social expresa cuán importante y apreciado es este asunto para la sociedad y sus representantes. La historia, estructura, calidad y financiamiento del sistema de salud, indican de alguna forma este sentido de prioridad y pertenencia social. Un problema conocido es la pobre calidad del gasto público en salud, es decir, eficiencia y transparencia con que se emplean los recursos públicos en las acciones y servicios de salud, lo que da espacio a la reticencia de grupos sociales y políticos para destinar recursos a la salud

La agenda estratégica presentada en este artículo no es prescriptiva. Es inapropiado dar recetas para alcanzar la universalidad con equidad en salud. Enfrentar con éxito los siete desafíos identificados y otros que cada país tenga requiere una buena dosis de decisión política, conocimientos, creatividad, motivación y dedicación. La historia de cada país, los valores predominantes en su cultura, el modelo político, económico y social y la evaluación de sus sistemas de salud, determinan cuál es el mejor camino. De hecho, no hay un solo camino ni un camino perfecto. 


\section{REFERENCIAS BIBLIOGRÁFICAS}

1. 26 Conferencia Sanitaria Panamericana. Extensión de la protección social en salud. Iniciativa conjunta. Washington: OPS, OIT; 2002.

2. WHA58.33. Sustainable health financing, universal coverage and social health insurance [sitio en Internet]. [citado 2009]. Disponible en:

http://www.who.int/healthsystems/topics /financing

3. Salud en Las Américas 2007. Washington, D.C.: OPS; 2007.

4. La aactual crisis financiera internacional y sus efectos en América Latina y EL Caribe. Santiago de Chile: CEPAL, Naciones Unidas; 2009.

5. WHO. Spending on health: a global overview [sitio en Internet]. [citado Mar 2007]. Disponible en: http://www.who.int/mediacentre

6. OMS. National Health Accounts [sitio en Internet]. [citado 2009]. Disponible en: http://www.who.int/nha/country/en/

7. Panorama Social de América Latina 2008. Santiago de Chile: CEPAL; 2008.

8. Informe sobre desarrollo humano 2007 2008. PNUD [sitio en Internet]. [citado 2009]. Disponible en: http://www.undp.org

9. Informe Sobre la Salud en el Mundo 2008. La Atención Primaria de la Salud, más necesaria que nunca. Ginebra: OMS; 2008.

10. Renovación de la atención primaria de salud en las Américas: documento de posición de la Organización Panamericana de la Salud/Organización Mundial de la Salud (OPS/OMS). Washington, D.C.: OPS/OMS; 2007.

11. Redes Integradas de Servicios de Salud. Conceptos y Opciones de Política y Hoja de Ruta para su implementación en Las Américas. Serie Renovación de la APA en las Américas. Washington, D.C.: OPS; 2008.

12. La Salud Pública en Las Américas. Nuevos conceptos, análisis del desempeño y bases para la acción. Washington, D.C.: OPS; 2008.

13. A gestão da saúde nos estados: avaliação e fortalecimento das funções esenciáis. Brasil: CONASS-OPAS; 2007.

14. Alianza Mundial por la Seguridad del Paciente. Ginebra: OMS; 2005.

15. Recursos humanos de salud en Las Américas: tendencias y datos para la acción. Unidad de Recursos Humanos. Washington, D.C.: OPS; 2006.

16. Figueras J. Health and wealth: assessing the case for investing in health systems. World Health Organization 2008 and World Health Organization, on behalf of the European Observatory on Health Systems and Policies. Geneva: WHO; 2008.

17. BLOG de la Directora, 16 de marzo de 2009. Washington, D.C. [sitio en Internet]. [citado 2009]. Disponible en: http://www.paho.org 
18. WHO. The World Medicines Situation. Geneva: WHO; 2004.

19. WHO. Equitable access to essential medicines: a framework for collective action. Geneva: WHO; 2004.

*Elaborado para el Primer Congreso de Medicina y Salud. UNAM, Ciudad de México, 22 a 24 de abril de 2009.

Recibido: 22 de marzo de 2009.

Aprobado: 4 de mayo de 2009.

Julio Manuel Suárez Jiménez. Consultor para el desarrollo de sistemas y servicios de salud, OPS/OMS. Avenida Dag Hammarskjöld 3269, Vitacura. Santiago de Chile. Chile. E-mail: juliosu@gmail.com 